\title{
Mathematical Modelling of Ultrasonic Non-Destructive Evaluation ${ }^{\dagger}$
}

\author{
LARISSA JU FRADKIN* \\ fradkil@sbu.ac.uk \\ Centre for Waves and Fields, School of EEIE, South Bank University, London SE 1 \\ $O A A$, England
}

VICTOR ZALIPAEV

zalipav@liverpool.ac.uk

Department of Mathematical Sciences, University of Liverpool, $M \& O$ Building, Peach Street, Liverpool L69 7ZL, United Kingdom

DMITRI GRIDIN

gridind@sbu.ac.uk

Centre for Waves and Fields, School of EEIE, South Bank University, London SE1 $O A A$, England

\begin{abstract}
High-frequency asymptotics have been used at our Centre to develop codes for modelling pulse propagation and scattering in the near-field of the ultrasonic transducers used in NDE (Non-Destructive Evaluation), particularly of walls of nuclear reactors. The codes are hundreds of times faster than the direct numerical codes but no less accurate.
\end{abstract}

\section{Introduction}

Our Centre specialises in mathematical modelling of NDE based on highfrequency asymptotics. We have been the first ${ }^{1-3}$ to produce the complete asymptotic description of the time-harmonic near field of a circular compressional transducer which is directly coupled to isotropic solid, both its geometrical regions and boundary layers in between the geometrical regions (see Fig. 1). Pulse propagation, rectangular transducers and transducers of complex apodisation have been also modelled using this approach ${ }^{4-6}$. The crux of the method is approximation of integrals containing an exponential factor, such that when observation point moves across the near field, the factor undergoes many oscillations while the amplitudes varies slowly. The main contributions to the integrals of this type come from the critical points: singularities of the amplitude, PSP (the stationary points

$\dagger$ Due to space limitation this article was omitted from the previous special issue on McNabb Symposium.

* Requests for reprints should be sent to Larissa Ju Fradkin, Centre for Waves and Fields, School of EEIE, South Bank University, London SE1 OAA, England 

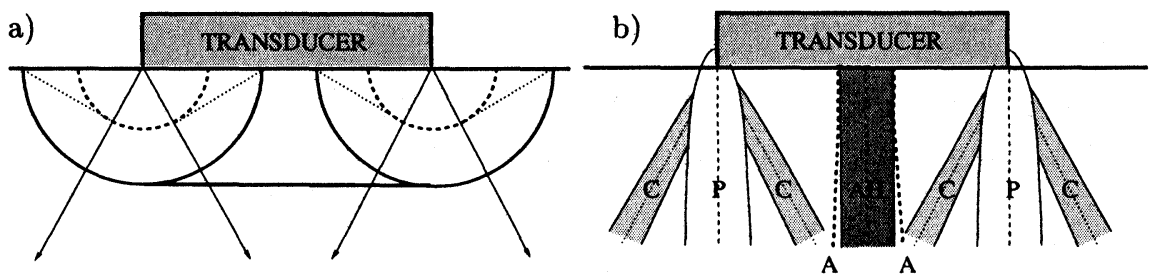

Figure 1. a) The wave fronts and b) boundary layers underneath a circular compressional transducer. Thick solid line - direct, thin solid lines - edge $P$, dashed lines - edge $S$, and dotted lines - edge head wave. $\mathrm{P}$ - penumbra, $\mathrm{AH}, \mathrm{AC}$ and zone between lines marked A - axial region, C - boundary layers around the critical rays.

of the phase functions) and various types of critical boundary points. Such contributions may be evaluated using various formulae of the USPM ${ }^{9-10}$ (Uniform Stationary Phase Method), depending on whether the critical points are isolated or coalesce. In the first case the exponential factor undergoes at least several oscillations in between the points, and in the second it does not. The resulting time-harmonic field contains the geometrical zones and boundary layers in between. The geometrical zones are described by ray-asymptotic series (in inverse powers of dimensionless wave number) which are contributions of isolated critical points. The boundary layers are described by boundary layer asymptotic series (in other types of functions of dimensionless wave number) which are due to coalescing critical points. We illustrate the approach in the next section.

\section{Ultrasonic modelling of an elliptic crack}

Let an elliptic crack be irradiated by a plane harmonic $\mathrm{P}$ wave $\mathbf{u}^{P(\text { inc })}=$ $\mathbf{n}^{P(i n c)} e^{i \mathbf{k}^{P(i n c)} \cdot \mathbf{x}}$, where (inc) stands for incident; $\alpha=P$ for compressional and $S$ for shear; wave number $k_{\alpha}=\omega / c_{\alpha}$, with $\omega$, the frequency and $c_{\alpha}$, the speed of $\alpha$-wave. Inside the solid, the total harmonic displacement field $\mathbf{u}=\mathbf{u}^{(\text {inc })}+\mathbf{u}^{\text {(scat) }}$, where (scat) stands for scattered is described by the reduced elastodynamic equation

$$
\left(c^{S}\right)^{2} \nabla \times(\nabla \times \mathbf{u})-\left(c^{P}\right)^{2} \nabla(\nabla \cdot \mathbf{u})-\omega^{2} \mathbf{u}=0 .
$$

On the boundary, the zero normal surface traction is assumed

$$
\sigma_{i z}=0 \text {, }
$$


where the total stress tensor $\sigma_{i j}=\lambda \delta_{i j} \cdot e_{i i}+2 \mu e_{i j} ; \delta_{i j}$ is the Kronecker delta; $\lambda$ and $\mu$ are the Lame constants; the total strain tensor is $e_{i j}=$ $\frac{1}{2}\left[u_{i, j}+u_{j, i}\right], i, j=x, y, z$ whatever the orientation of $x$ - and $y$-axis; index before the comma denotes a component, and index after the comma denotes differentiation with respect to the corresponding spatial variable. The usual radiation condition at infinity and Meixner's condition at the edge are assumed. We can use the well-known scalar and vector potentials, $\psi_{0}$ and $\boldsymbol{\psi}=\left(\psi_{1}, \psi_{2}, \psi_{3}\right)$, respectively and write

$$
\mathbf{u}^{P}=\nabla \psi_{0}, \quad \mathbf{u}^{S}=\nabla \times \boldsymbol{\psi}
$$

First let us recall the

\subsection{Canonical problem}

Let a plane compressional wave scatter off a semi-infinite crack. Let us introduce the standard Cartesian coordinate system with the $\mathrm{x}$-axis running along the edge of the crack and the $y$-axis directed into the crack half-plane. The corresponding spherical coordinates are $(r, \phi, \theta)$. Then the unit wave vector $\mathbf{n}^{P(\text { inc })}$ is

$$
\mathbf{n}^{P(i n c)}=\left(\sin \theta^{(i n c)} \cos \varphi^{(i n c)}, \sin \theta^{(i n c)} \sin \varphi^{(i n c)},-\cos \theta^{(i n c)}\right) .
$$

Without loss of generality let us assume that the incident wave propagates from above. The problem has been studied by many authors ${ }^{7}$. We shall employ respective even and odd parts of the potentials of the scattered field as derived in Achenbach et al. ${ }^{7}$,

$$
\begin{aligned}
\psi_{\ell}^{+(c a n)}(\mathbf{x}) & =\frac{e^{i \eta x}}{2 \pi} \int_{-\infty}^{+\infty} \frac{A_{\ell}^{+}(\xi)}{\gamma_{\alpha}(\xi)\left(\xi+\xi^{i n c}\right)} e^{-i \xi y+i \gamma_{\alpha}(\xi)|z|} d \xi, \\
\psi_{\ell}^{-(c a n)}(\mathbf{x}) & =\operatorname{sgn}(z) \frac{e^{i \eta x}}{2 \pi} \int_{-\infty}^{+\infty} \frac{A_{\ell}^{-}(\xi)}{\xi+\xi^{i n c}} e^{-i \xi y+i \gamma_{\alpha}(\xi)|z|} d \xi, \\
\alpha & = \begin{cases}P, & \text { if } \ell=0, \\
S, & \text { if } \ell=1,2,3,\end{cases}
\end{aligned}
$$

where a total potential component is $\psi_{\ell}^{(\text {can })}(\mathbf{x})=\psi_{\ell}^{+(c a n)}(\mathbf{x})+\psi_{\ell}^{-(c a n)}(\mathbf{x})$. The descriptor (can) refers to the canonical problem; the projection of the incident wave-vector $\mathbf{k}^{P(\text { inc })}$ onto the $\mathrm{x}$-axis is $\eta=k_{x}^{P(\text { inc })}$. Equations (5) represent potentials as integrals over plane waves $\exp \left(i \mathbf{k}^{\alpha} \cdot \mathbf{x}\right)$ with complex wave vectors

$$
\mathbf{k}^{\alpha}=\left(\eta,-\xi, \operatorname{sgn}(z) \gamma_{\alpha}(\xi)\right)
$$


so that we have $\gamma_{\alpha}(\xi)=\sqrt{k_{\alpha}^{2}-\eta^{2}-\xi^{2}}$. The functions $A_{\ell}^{ \pm}(\xi)$ are regular in the upper half $\xi$-plane and are given in Appendix A. The corresponding scalar and vector potentials involve combinations

$$
A_{\ell}(\xi)=A_{\ell}^{+}(\xi)+\operatorname{sgn}(z) \frac{\gamma_{\alpha}(\xi)}{k_{S}} A_{\ell}^{-}(\xi)
$$

where $\mathbf{e}_{\ell}=\mathbf{e}_{x}, \mathbf{e}_{y}$ or $\mathbf{e}_{z}$ for $\ell=1,2$ or 3 respectively. Using (3) the respective displacements involve vectors

$$
A^{P}(\xi) \mathbf{n}^{P}(\xi)=A_{0}(\xi) \mathbf{n}^{P}(\xi), \quad A^{S}(\xi) \mathbf{n}_{\perp}^{S}(\xi)=\sum_{\ell=1}^{3} A_{\ell}(\xi) \mathbf{n}^{S}(\xi) \times \mathbf{e}_{\ell}
$$

where $A^{S}$ and $\mathbf{n}_{\perp}^{S}$ are both defined by the above formula. The functions have a pole

$$
-\xi^{i n c}=-k_{y}^{P(i n c)} .
$$

The integration contour in (5) passes it from above. The other critical points are

$$
\xi^{\alpha(G T D)}=-\sqrt{k_{\alpha}^{2}-\eta^{2}} \frac{y}{\sqrt{y^{2}+z^{2}}} .
$$

The descriptor (GTD) in (10) is justified below. For simplicity of presentation, we consider the scattered field only in the upper half-space $z>0$.

If the observation point in the upper half-space is such that the pole (9) and the phase stationary points (10) are isolated from each other, we get two contributions:

\section{The contribution of the isolated pole: $G E$ reflected waves}

Applying to (5) the standard formulae for the pole contribution (Borovikov ${ }^{9}$, 1994, Sec. 1.6) and using (3), the leading order terms are

$$
\mathbf{u}^{\alpha(G E)}(\mathbf{x})=H\left[y-z \frac{k_{y}^{\alpha(r e f)}}{k_{z}^{\alpha(r e f)}}\right] R^{\alpha} \mathbf{p}^{\alpha(r e f)} e^{i \mathbf{k}^{\alpha(r e f)} \cdot \mathbf{x}},
$$

where $H(x)$ is a Heaviside Function, the reflected wave vector is

$$
k^{\alpha(r e f)}=\left(k_{x}^{P(\text { inc })}, k_{y}^{P(\text { inc })}, \sqrt{\left.k_{\alpha}^{2}-\left(k_{x}^{\alpha(r e f)}\right)^{2}-\left(k_{y}^{\alpha(r e f)}\right)^{2}\right)} .\right.
$$

Above, the unit reflected wave vector is $\mathbf{n}^{\alpha(r e f)}=\left(\sin \theta^{\alpha(r e f)} \cos \varphi^{(r e f)}\right.$, $\left.\sin \theta^{\alpha(r e f)} \sin \varphi^{(r e f)}, \cos \theta^{\alpha(r e f)}\right) ; R_{P}$ and $R_{S}$ are standard reflection coefficients ${ }^{7}$, 
and the respective polarisation vectors $\mathbf{p}^{P(r e f)}=\mathbf{n}^{P(r e f)}$ and $\mathbf{p}^{S(r e f)}=$ $\mathbf{n}_{\perp}^{S(r e f)}$, with the unit vector of S-displacement being $\mathbf{n}_{\perp}^{S(r e f)}=\left(-\cos \theta^{S(r e f)} \cos \varphi^{(r e f)}\right.$, $\left.-\cos \theta^{S(r e f)} \sin \varphi^{(r e f)}, \sin \theta^{S(r e f)}\right)$. Combining (4) and (12) it follows that the above angles are defined by

$$
k_{\alpha} \sin \theta^{\alpha(r e f)}=k_{P} \sin \theta^{(i n c)}, \quad \varphi^{\alpha(r e f)}=\varphi^{\alpha(i n c)} .
$$

Let us now consider a point $\mathbf{x}^{\alpha(r e f)}=\left(x^{\alpha(r e f)}, y^{\alpha(r e f)}, 0\right)$ lying in the plane of the crack, such that we have

$$
k^{\alpha(r e f)}=\frac{k_{\alpha}}{d^{\alpha(r e f)}}\left(\mathbf{x}-\mathbf{x}^{\alpha(r e f)}\right),
$$

where distance $d^{\alpha(r e f)}=\left|\mathbf{x}-\mathbf{x}^{\alpha(r e f)}\right|$. This vector equation results in two scalar equations in two unknowns, $x^{\alpha(r e f)}$ and $y^{\alpha(r e f)}$. It follows that we have

$$
x^{\alpha(r e f)}=x-z \frac{k_{x}^{\alpha(r e f)}}{k_{z}^{\alpha(r e f)}}, y^{\alpha(r e f)}=y-z \frac{k_{y}^{\alpha(r e f)}}{k_{z}^{\alpha(r e f)}}, \quad d^{\alpha(r e f)}=\frac{z}{\cos \theta^{\alpha(r e f)}} .
$$

It is clear that if $y=z k_{y}^{\alpha(r e f)} / k_{z}^{\alpha(r e f)}$, we get $y^{\alpha(r e f)}=0$, that is $\mathbf{x}^{\alpha(r e f)}$ lies on the crack edge. Thus, the argument of the Heaviside function in (11) is positive when $\mathbf{x}^{\alpha(r e f)}$ lies on the crack, and negative when it lies in the same plane as, but outside, the crack; in both cases the formula applies only when the observation point lies far from the edge. Note that using (12) and (14), the total phase may be decomposed into incident and reflected as follows:

$$
k^{\alpha(r e f)} \cdot \mathbf{x}=\mathbf{k}^{P(i n c)} \cdot \mathbf{x}^{\alpha(r e f)}+k_{\alpha} d^{\alpha(r e f)} .
$$

Thus, the incident ray that hits this point gives rise to the reflected ray which reaches $\mathbf{x}$. In the geometrical acoustics such points are called specular.

It follows that the formula (11) describes the well-known main reflected $\alpha$-beams, and their geometrical shadows. The beams obey the Snell's law (13). Note also that neither the position of $\mathbf{x}^{\alpha(r e f)}$ nor the contribution (11) depend on the position or orientation of the crack edge, and (11) constitutes an exact solution of the problem of reflection from an infinite plane. 


\section{The contribution of the isolated phase stationary point: GTD edge diffracted waves}

To simplify the formulae below, let us introduce instead of $(r, \varphi, \theta)$, the spherical coordinates $(r, \vartheta, \Omega)$ corresponding to the Cartesian system $\left(\mathbf{e}_{y}, \mathbf{e}_{z}, \mathbf{e}_{x}\right)$. Applying to (5) the standard asymptotic formula of the stationary phase method (Borovikov ${ }^{9}, 1994$, Sec. 1.2) and using (3), to the leading order in $k_{\alpha} r$ we obtain

$\mathbf{u}^{\alpha(G T D)}(\mathbf{x})=\frac{D^{\alpha}}{\sqrt{k_{\alpha} J^{\alpha}}} \cdot \mathbf{p}^{\alpha(G T D)} \cdot e^{i \mathbf{k}^{\alpha(G T D)} \cdot \mathbf{x}+i \pi / 4}, \quad\left|y-\cot \vartheta^{\alpha(r e f)} \cdot z\right|>0$,

where $\mathbf{k}^{\alpha(G T D)}=\left(k_{x}^{P(\text { inc })}, k_{\alpha} \sin \Omega^{\alpha(G T D)} \cos \vartheta, k_{\alpha} \sin \Omega^{\alpha(G T D)} \sin \vartheta\right) ; \Omega^{\alpha}$ is the angle between wave vector $\mathbf{k}^{\alpha}$ and the $x$-axis; and the gradient of the geometrical $\alpha$-shadow boundary is given by $\cot \vartheta^{\alpha(r e f)}=k_{y}^{\alpha(r e f)} / k_{z}^{\alpha(r e f)}$. It follows that we have

$$
k_{\alpha} \cos \Omega^{\alpha(G T D)}=k_{P} \cos \Omega^{P(i n c)}
$$

Note that using (10) and (12), we also have

$$
\xi^{\alpha}=-k_{y}^{\alpha(G T D)} \text {. }
$$

The conditions in (17) are refined below in the section on the penumbral contribution. To continue, the diffraction coefficients in (17) are

$$
D^{\alpha}=\frac{A^{\alpha}\left(-k_{y}^{\alpha(G T D)}\right)}{\sqrt{2 \pi} \sin \Omega^{\alpha(G T D)}\left(\cos \vartheta^{\alpha(r e f)}-\cos \vartheta^{\alpha}\right)},
$$

the geometrical spreading is

$$
J^{\alpha}=\sqrt{y^{2}+z^{2}} \sin \Omega^{\alpha(G T D)} ;
$$

and the respective polarisation vectors are $\mathbf{p}^{P(G T D)}=\mathbf{n}^{P(G T D)}$ and $\mathbf{p}^{S(G T D)}=$ $\mathbf{n}_{\perp}^{S(r e f)}\left(-k_{y}^{\alpha(G T D)}\right)$.

Let us now consider a point $\mathbf{x}^{\alpha(G T D)}=\left(x^{\alpha(G T D)}, 0,0\right)$ lying on the edge of the crack, such that we have

$$
\mathbf{k}^{\alpha(G T D)}=\frac{k_{\alpha}}{d^{\alpha(G T D)}}\left(\mathbf{x}-\mathbf{x}^{\alpha(G T D)}\right) .
$$

The above vector equation results in one scalar equation for $x^{\alpha(G T D)}$. Thus, we have

$$
x^{\alpha(G T D)}=x-\sqrt{y^{2}+z^{2}} \cot \Omega^{\alpha(G T D)}, \quad d^{\alpha(G T D)}=\frac{\sqrt{y^{2}+z^{2}}}{\sin \Omega^{\alpha(G T D)}} .
$$


Thus, an incident ray that hits this point gives rise to a family of diffracted rays lying on the surface of a cone with the tip at $\mathrm{x}^{\alpha(G T D)}$, the axis running along the $\mathrm{x}$-axis and half-angle $\Omega^{\alpha(G T D)}$ which are related to $\Omega^{P(\text { inc })}$ by the Snell's law of diffraction (18). In GTD such points are known as flash points. Note also that the total phase may be decomposed into incident and diffracted as follows:

$$
\mathbf{k}^{\alpha(G T D)} \cdot \mathbf{x}=\mathbf{k}^{P(i n c)} \cdot \mathbf{x}^{\alpha(G T D)}+k_{\alpha} d^{\alpha(G T D)} .
$$

It follows that (17) describes the well-known in GTD edge diffracted waves.

To summarise, in the geometrical regions the high-frequency asymptotics of the scattered field are represented by a sum of the first terms in the GE and GTD ray series ${ }^{1}$

$$
\mathbf{u}^{\alpha(c a n)}(\mathbf{x}) \sim \mathbf{u}^{\alpha(G E)}(\mathbf{x})+\mathbf{u}^{\alpha(G T D)}(\mathbf{x}),
$$

where $\mathbf{u}^{\alpha(G E)}(\mathbf{x})$, zero in the shadows, is independent of the the location and orientation of the edge and $\mathbf{u}^{\alpha(G T D)}(\mathbf{x})$ is a term of a higher order in dimensionless frequency.

\section{The coalescing pole and stationary phase point: penumbra}

When the observation point is such that the pole (9) and the phase stationary point in (10) coalesce, applying to (5) the corresponding asymptotic formula of the stationary phase method (Borovikov ${ }^{9}, 1994$, Eq. (2.28)), the leading order contribution is

$$
\mathbf{u}^{\alpha(p e n)}(\mathbf{x})=F\left(v_{\alpha}\right) \mathbf{u}^{\alpha(G E)}(\mathbf{x})+\frac{\tilde{D}^{\alpha}}{\sqrt{k_{\alpha} J^{\alpha}}} \cdot \mathbf{p}^{\alpha(G T D)} e^{i \mathbf{k}^{\alpha(G T D)} \cdot \mathbf{x}+i \pi / 4}(25)
$$

where $\left(v^{\alpha}\right)^{2} \leq \pi$, the first term in the right-hand side is expressed as usual via the Fresnel integral $F(v)=(\pi i)^{-1 / 2} \int_{v}^{\infty} \exp \left(i t^{2}\right) d t$ with

$$
v_{\alpha}=\operatorname{sgn}\left[\vartheta-\vartheta^{\alpha(\mathrm{ref})}\right] \sqrt{\left(\mathrm{k}^{\alpha(\mathrm{GTD})}-\mathrm{k}^{\alpha(\mathrm{ref})}\right) \cdot \mathbf{x}}
$$

and the modified diffraction coefficients $\tilde{D}^{\alpha}$ as in the formulae (20) but with $A_{\ell}\left(\xi^{\alpha}\right)$ replaced by $A_{\ell}\left(\xi^{\alpha}\right)-A_{\ell}\left(-\xi^{i n c}\right)$. It is clear that $v_{\alpha}^{2}$ is the difference between the phases of the diffracted edge and reflected ray reaching the observation point $\mathbf{x}$. The coalescence of the pole (9) with the phase stationary point (10), that is the coalescence of the corresponding specular and flash point occurs when the observation point $\mathbf{x}$ crosses a geometrical $\alpha$-shadow boundary and thus the reflected ray coalesces with the diffracted ray to form the so-called marginal ray, $\vartheta=\vartheta^{\alpha(r e f)}$, on the 
diffraction cone. The transition zone (boundary layer) surrounding this boundary is known as penumbra. As usual, it is defined by $\left(v^{\alpha}\right)^{2} \leq \pi$, so that inside it the phase difference is less than $\pi$ and the exponential factor in (5) ceases to be rapidly oscillating. To find the boundary of penumbral region we note that using $(18) k^{\alpha(r e f)}$ can be re-written as $k^{\alpha(r e f)}=$ $\left(k_{\alpha} \cos \Omega^{\alpha(G T D)}, k_{\alpha} \sin \Omega^{\alpha(G T D)} \cos \vartheta^{\alpha(r e f)}, k_{\alpha} \sin \Omega^{\alpha(G T D)} \sin \vartheta^{\alpha(r e f)}\right)$. Therefore,

combining the above formula with (12), the equation of the penumbra boundary becomes $2 k_{\alpha} \sqrt{y^{2}+z^{2}} \sin \Omega^{\alpha(G T D)} \sin ^{2}\left(\vartheta-\vartheta^{\alpha(r e f)}\right) / 2=\pi$, which is the equation of a parabolic cylindrical surface surrounding the plane geometrical $\alpha$-shadow boundary. The corresponding conditions in (11), (17) and (25) may be refined accordingly to specify that these formulae apply inside or outside the penumbral region respectively.

Evaluating asymptotics of (25) when $v_{\alpha} \rightarrow \infty$ we obtain the behaviour which is entirely consistent with the expected physical picture: the GE reflected and edge diffracted $\mathrm{P}$ and $\mathrm{S}$ waves in the main beam zone and just edge diffracted $\mathrm{P}$ and $\mathrm{S}$ waves in the geometrical shadow zone.

To summarise, in the penumbra the high-frequency asymptotics of the scattered field may be represented by the first two terms of the penumbral series, $\mathbf{u}^{\alpha(p e n)}(\mathbf{x})$.

\subsection{Scattering of a plane $P$ wave from a planar crack with an elliptic edge}

Let us now consider scattering from an elliptic crack. Let the new Cartesian coordinate system be $(x, y, z)$, with the origin at the ellipse centre, the $\mathrm{x}$ axis running along the crack major semi-axis and the $y$-axis, along its minor axis. Let the corresponding spherical polar system be again $(r, \varphi, \theta)$. The parametric equations of the elliptic crack edge are $x^{\prime}(\sigma)=a \cos \sigma, \quad y^{\prime}(\sigma)=$ $b \sin \sigma$, with the semi-axes $a \geq b$ and parameter $\sigma \in[0,2 \pi]$.

We proceed by deriving the radiating near field asymptotics $\left(r \gg\left(k_{P}\right)^{-1}\right.$ and $r \ll k_{P} a b$.) We start by writing the decoupled form of the classical Green's formula

$\psi_{\ell}^{+}(\mathbf{x})=\int_{D} G_{\alpha}^{+}\left(\mathbf{x}, \mathbf{x}^{\prime}\right) \frac{\partial \psi_{\ell}^{+}}{\partial z^{\prime}}\left(\mathbf{x}^{\prime}\right) d x^{\prime} d y^{\prime}, \psi_{\ell}^{-}(\mathbf{x})=-\int_{D} \frac{\partial G_{\alpha}^{-}}{\partial z^{\prime}}\left(\mathbf{x}, \mathbf{x}^{\prime}\right) \psi_{\ell}^{-}\left(\mathbf{x}^{\prime}\right) d x^{\prime} d y^{\prime}$

where the region $D$ is the crack face; $\partial / \partial z^{\prime}$ is a derivative with respect to the outward normal to the crack face; $\psi_{\ell}^{ \pm}(\mathbf{x})$ and $G_{\alpha}^{ \pm}\left(\mathbf{x}, \mathbf{x}^{\prime}\right)$ are even and 


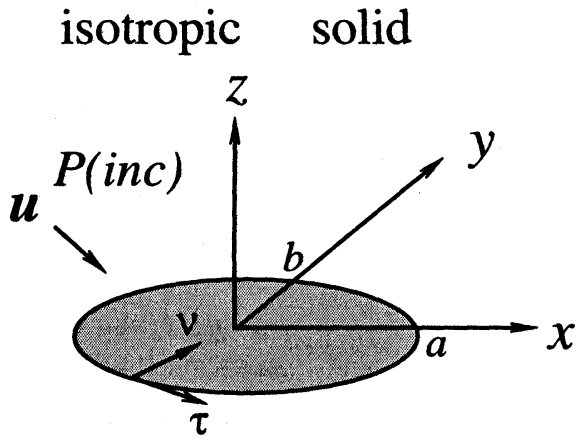

Figure 2. Scattering from an elliptic crack.

odd combinations related to $\psi_{\ell}(\mathbf{x})$ and $G_{\alpha}\left(\mathbf{x}, \mathbf{x}^{\prime}\right)$ respectively, namely we have

$$
\begin{gathered}
\psi_{\ell}^{ \pm}(\mathbf{x})=\frac{\psi_{\ell}(x, y, z) \pm \psi_{\ell}(x, y,-z)}{2} \\
G_{\alpha}^{ \pm}\left(\mathbf{x}, \mathbf{x}^{\prime}\right)=G_{\alpha}\left(x, y, z ; x^{\prime}, y^{\prime}, z^{\prime}\right) \pm G_{\alpha}\left(x, y,-z ; x^{\prime}, y^{\prime}, z^{\prime}\right) ;
\end{gathered}
$$

and the free space Green's function is

$$
G_{\alpha}\left(\mathbf{x} ; \mathbf{x}^{\prime}\right)=-\frac{e^{i k_{\alpha} d^{\prime}}}{4 \pi d^{\prime}}
$$

with distance $d^{\prime}=\left|\mathbf{x}-\mathbf{x}^{\prime}\right|$. In the leading order in $k_{\alpha} d^{\prime}$, we have

$$
\frac{\partial G_{\alpha}^{-}}{\partial z^{\prime}}\left(\mathbf{x}, \mathbf{x}^{\prime}\right) \approx \frac{i k_{\alpha} z e^{i k_{\alpha} d^{\prime}}}{2 \pi d^{\prime 2}}
$$

Let us evaluate (26) by using the two-tier asymptotics ${ }^{1}$.

\subsubsection{First tier}

In order to build the first tier asymptotics, we first note that in the canonical case, when the pole $\xi^{i n c}$ is isolated, and hence the specular points $\mathbf{x}^{\alpha(r e f)}$ are far from the edge, on substituting (5) into (26) the resulting integrals may be approximated by contributions of $\mathbf{x}^{\alpha(r e f)}$ and the crack edge. These contributions may be obtained using a separation of unity $\left\{\chi_{1}, \chi_{2}, \chi_{3}\right\}$, where the neutraliser $\chi_{1}$ has support in the neighbourhood of $\mathbf{x}^{\alpha(r e f)} ; \chi_{2}$, in the neighbourhood of the crack edge, and $\chi_{3}=1-\chi_{1}-\chi_{2}$. Therefore, when scattering from the elliptic crack, we can use 
Assumption 1: When a specular point $\mathbf{x}^{\alpha(s p e c)}$ is far from the ellipse edge, the potentials may be approximated as follows

$$
\psi_{\ell}^{s c a t}(\mathbf{x}) \sim \psi_{\ell}^{(G E)}(\mathbf{x})+\hat{\psi}_{\ell}^{(\operatorname{diff})}(\mathbf{x}),
$$

and, when the specular point is near the ellipse edge, we have

$$
\psi_{\ell}^{\text {scat }}(\mathbf{x}) \sim \hat{\psi}_{\ell}^{(\operatorname{diff})}(\mathbf{x})
$$

Since, the expressions in (11) do not depend on the position or location of the crack boundary either, $\psi_{\ell}^{(G E)}$ are such that we have

$$
\mathbf{u}^{\alpha(G E)}(\mathbf{x})=H\left[1-\frac{\left(x^{\alpha(r e f)}\right)^{2}}{a^{2}}-\frac{\left(y^{\alpha(r e f)}\right)^{2}}{b^{2}}\right] R^{\alpha} \mathbf{p}^{\alpha(r e f)} e^{i \mathbf{k}^{\alpha(r e f)} \cdot \mathbf{x}}
$$

We remind the reader that in this paper we present results only for $z>0$. On the other hand, the modified diffracted fields in (31) and (32) are to the leading order in $k_{\alpha} r$, contributions to the following integrals

$$
\begin{aligned}
& \hat{\psi}_{\ell}^{+(d i f f)}(\mathbf{x})=2 \int_{D} \chi_{2}\left(\mathbf{x}^{\prime}\right) G_{\alpha}^{+}\left(\mathbf{x}, \mathbf{x}^{\prime}\right) \frac{\partial \psi_{\ell}^{+}}{\partial z^{\prime}}\left(\mathbf{x}^{\prime}\right) d x^{\prime} d y^{\prime}, \\
& \hat{\psi}_{\ell}^{-(d i f f)}(\mathbf{x})=-2 \int_{D} \chi_{2}\left(\mathbf{x}^{\prime}\right) \frac{\partial G_{\alpha}^{-}}{\partial z^{\prime}}\left(\mathbf{x}, \mathbf{x}^{\prime}\right) \psi_{\ell}^{-}\left(\mathbf{x}^{\prime}\right) d x^{\prime} d y^{\prime},
\end{aligned}
$$

where $D$ is the elliptic crack. The contributions implied in (31) and (32) are different, since they depend on the position of the specular point. We evaluate them approimately with the help of often used

Assumption 2 (The localisation principle): In the high-frequency approximation we assume that near the crack edge, to the leading order in $k_{P} \rho_{\min }$, the leading terms of the scattered field are the same as in the corresponding canonical problem, with the edge of the semi-infinite crack tangent to the elliptic crack edge at this point.

For convenience, in the neighbourhood of the edge we employ local coordinates $(\sigma, \nu)$, where $\nu$ is the distance along the internal normal to the edge along, with the two unit vectors $\mathbf{e}_{\tau}(\sigma)$ and $\mathbf{e}_{\nu}(\sigma)$ tangent and normal to the ellipse edge correspondingly. Below we do not mention $\sigma$-dependance unless absolutely necessary. Then we can approximate the crack values of the potentials (5) by assuming that at each point $\mathbf{x}^{\prime}=(\sigma, \nu)$ the phase of each plane wave in the superposition may be decomposed into incident and scattered, $\mathbf{k}^{P(\text { inc })} \cdot \mathbf{x}^{\prime}(\sigma)-\xi \nu$, where $\mathbf{x}^{\prime}(\sigma)$ is projection of $\mathbf{x}^{\prime}$ onto the crack 
edge. Then on the irradiated crack side we have

$$
\begin{aligned}
\frac{\partial \tilde{\psi}_{\ell}^{+}}{\partial z^{\prime}}(\sigma, \nu) & =\frac{i k_{\alpha} e^{i \mathbf{k}^{P(i n c)} \cdot \mathbf{x}^{\prime}(\sigma)}}{2 \pi} \int_{-\infty}^{+\infty} \frac{A_{\ell}^{+}\left(k_{\alpha} \zeta\right) e^{-i k_{\alpha} \zeta \nu}}{\zeta+\zeta^{r e f}(\sigma)} d \zeta, \\
\tilde{\psi}_{\ell}^{-}(\sigma, \nu) & =\frac{k_{\alpha} e^{i \mathbf{k}^{P(i n c)} \cdot \mathbf{x}^{\prime}(\sigma)}}{2 \pi} \int_{-\infty}^{+\infty} \frac{A_{\ell}^{-}\left(k_{\alpha} \zeta\right) e^{-i k_{\alpha} \zeta \nu}}{\zeta+\zeta^{r e f}(\sigma)} d \zeta
\end{aligned}
$$

where we assume the pole of the integrand to be

$$
-\xi^{i n c}(\sigma)=-\mathbf{n}^{P(i n c)} \cdot \mathbf{e}_{\nu}(\sigma) .
$$

Note that in this approximation the components $\partial \tilde{\psi}_{\ell}^{+} / \partial z^{\prime}(\sigma, n)$ and $\tilde{\psi}_{\ell}^{-}(\sigma, n)$ are given when applicable in the local base $\left(\mathbf{e}_{\tau}(\sigma), \mathbf{e}_{\nu}(\sigma), \mathbf{e}_{z}\right)$. The non-zero elements of the transition matrix $T$ from this base to $\left\{\mathbf{e}_{x}, \mathbf{e}_{y}, \mathbf{e}_{z}\right\}$ are given as

$$
T_{11}=T_{22}=\mathbf{e}_{\tau}(\sigma) \cdot \mathbf{e}_{x}, T_{12}=-T_{21}=\mathbf{e}_{\tau}(\sigma) \cdot \mathbf{e}_{y}, \quad T_{33}=1
$$

By substituting (35) into (34), we obtain the following triple integral

$$
\begin{aligned}
& \hat{\psi}_{\ell}^{(d i f f)}(\mathbf{x}) \approx-\frac{i k_{\alpha}}{(2 \pi)^{2}} \int_{0}^{2 \pi} d \sigma \int_{0}^{\infty} d \nu \int_{-\infty}^{\infty} d \zeta \chi_{2}(\nu)\left[1-\frac{\nu}{\rho(\sigma)}\right] \frac{q(\sigma)}{d^{\prime}} \\
& \left.\left\{\sum_{l^{\prime}=0}^{3} \frac{T_{\ell^{\prime} \ell}(\sigma)}{\zeta+\zeta^{r e f}(\sigma)}\left[A_{\ell^{\prime}}^{+}\left(k_{\alpha} \zeta\right)+\frac{k_{\alpha} z}{d^{\prime}} A_{\ell^{\prime}}^{-}\left(k_{\alpha} \zeta\right)\right]\right\} e^{i \mathbf{k}^{P(\text { inc })} \cdot \mathbf{x}^{\prime}(\sigma)+i k_{\alpha}\left[d^{\prime}\right.}(\xi) \mathcal{8}\right)
\end{aligned}
$$

where the matrix $T_{\ell^{\prime} \ell}$ is extended to $4 \times 4$ matrix, with the only new nonzero element $T_{00}=1 ; q(\sigma)=\sqrt{(a \sin \sigma)^{2}+(b \cos \sigma)^{2}}$; and $[1-\nu / \rho(\sigma)]$ is the Jacobian of the transformation from $(x, y)$ to $(\sigma, \nu)$.

\subsubsection{Second Tier}

The critical points in (38) are an amplitude pole, PSP and boundary critical points of the second kind ${ }^{10}$. Let us consider their contributions in turn.

Contribution of the isolated critical boundary points: GTD edge diffracted waves The PSPs of the double integral in $(\nu, \zeta)$ in $(38)$ are

$$
\nu=0, \quad \zeta^{\alpha}=-\frac{\left(\mathbf{x}-\mathbf{x}^{\prime}(\sigma)\right) \cdot \mathbf{e}_{\nu}}{d^{\prime}},
$$


where $d^{\prime}=\sqrt{\left(x-x^{\prime}(\sigma)\right)^{2}+\left(y-y^{\prime}(\sigma)\right)^{2}+z^{2}}$. Let a critical point like this be isolated. Then applying stationary phase method (SPM), we get the Edge Integral

$$
\hat{\mathbf{u}}^{\alpha(G T D)}(\mathbf{x}) \sim \int_{0}^{2 \pi} \frac{\mathrm{B}^{\alpha}}{d^{\prime}} \mathrm{e}^{i k_{\alpha} d^{\alpha(t o t)}} d \sigma
$$

where $k_{\alpha} d^{\alpha(\text { tot })}=\mathbf{k}^{P(\text { inc })} \cdot \mathbf{x}^{\prime}(\sigma)+k_{\alpha} d^{\prime}$; and the diffracted directivity patterns are

$$
\mathbf{B}^{\alpha}(\sigma)=\frac{q(\sigma)}{2 \pi} \frac{A^{\alpha}\left(k_{\alpha} \zeta(\sigma)\right)}{\left[\mathbf{n}^{\alpha(r e f)}-\mathbf{n}(\sigma)\right] \cdot \mathbf{e}_{\nu}(\sigma)} \mathbf{p}^{\alpha}(\sigma),
$$

with the polarisation vectors $\mathbf{p}^{P}=\mathbf{n}(\sigma)$ and $\mathbf{p}^{S}=\mathbf{n}_{\perp}^{S}(\sigma)$. McMaken ${ }^{8}$ derived an analogous edge integral for normal incidence of plane $\mathrm{P}$-wave on a penny-shaped crack.

The boundary critical points, $\sigma_{m}^{\alpha}$, in (38) are PSPs of the Edge Integral which may be found numerically. Their number $M_{\alpha}$ may be 2 to 4 . Let all $\sigma_{m}^{\alpha}$ be isolated. Then applying SPM to the Edge Integral, we have

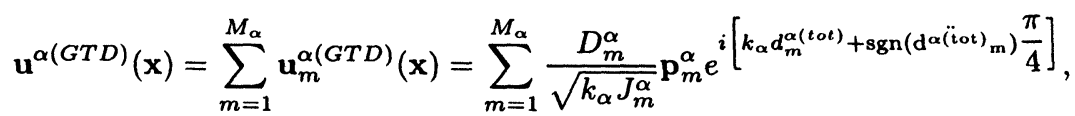

where $\mathbf{u}_{m}^{\alpha(G T D)}$ are each described by a formula similar to (17), with all $\left(\begin{array}{l}\alpha \\ m\end{array}\right)$ quantities evaluated at $\sigma=\sigma_{m}^{\alpha}$. The above formula is in agreement with GTD. Inside each $\alpha$-geometrical region, the $\alpha$-component of the scattered field is $\mathbf{u}^{\alpha(\text { scat })}=\mathbf{u}^{\alpha(G E)}+\sum_{m=1}^{M_{\alpha}} \mathbf{u}_{m}^{\alpha(G T D)}$.

\section{Coalescing amplitude pole and a PSP: Penumbras of the scattered field}

A formula similar to (25) can be re-derived in the framework of the two-tier appraoch, with the result that $\mathbf{k}^{\alpha(G T D)}$ is replaced by $\mathbf{k}_{1}^{\alpha(G T D)}, \mathbf{p}^{\alpha(G T D)}$ by $\mathrm{p}_{1}^{\alpha(G T D)}$ etc. The coalescence of the specular point $\mathrm{x}^{\alpha(r e f)}$ with one of the flash points $\mathbf{x}_{1}^{\alpha(G T D)}$ corresponds to observation point $\mathbf{x}$ crossing a geometrical shadow boundary. Inside each $\alpha$-penumbra, the $\alpha$-component of the scattered field is $\mathbf{u}^{\alpha(\text { scat })}=\mathbf{u}_{1}^{\alpha(p e n)}+\sum_{m=2}^{M_{\alpha}} \mathbf{u}_{m}^{\alpha(G T D)}$.

\section{Coalescing critical boundary points: Caustic regions}

Let $\sigma_{1}^{\alpha}$ and $\sigma_{2}^{\alpha}$ in Edge Integral coalesce. Applying USPM ${ }^{9,10}$, we have

$$
\mathbf{u}_{1,2}^{\alpha(\text { caus })}=\frac{\pi \sqrt{2}}{k_{\alpha}^{1 / 3}}\left\{\mathbf{C}_{+}^{\alpha} A i\left[-k_{\alpha}^{2 / 3} \mu_{\alpha}\right]+\mathbf{C}_{-}^{\alpha} \frac{A i^{\prime}\left[-k_{\alpha}^{2 / 3} \mu_{\alpha}\right]}{i k_{\alpha}^{1 / 3}}\right\} \mathrm{e}^{i k_{\alpha} d^{\alpha}}
$$


(a)

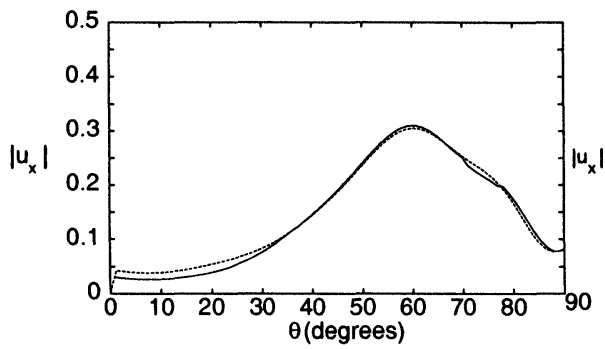

(b)

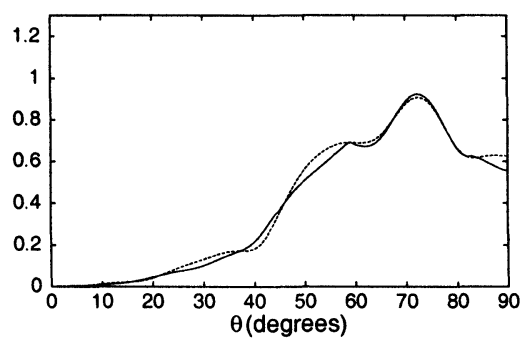

Figure 3. Asymptotic solution (solid line) vs numerical solution (dashed line), $\theta^{(\text {ref })}=$ $30^{\circ}, \varphi^{(r e f)}=45^{\circ}, a=4 \mathrm{~mm}, b=2 \mathrm{~mm}, r=5 \mathrm{~mm}, \varphi=45^{\circ}$, (a) $\mathrm{P}$ wave, (b) $\mathrm{S}$ wave.

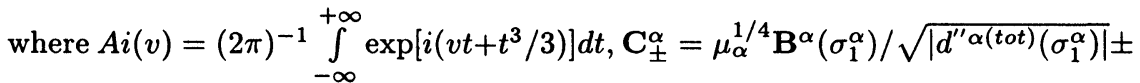
$\mathbf{B}^{\alpha}\left(\sigma_{2}^{\alpha}\right) / \mu_{\alpha}^{1 / 4} \sqrt{\left|d^{\prime \prime \alpha(t o t)}\left(\sigma_{2}^{\alpha}\right)\right|}, d^{\alpha}=\left[d^{\alpha(t o t)}\left(\sigma_{1}^{\alpha}\right)+d^{\alpha(t o t)}\left(\sigma_{2}^{\alpha}\right)\right] / 2$ and $\mu_{\alpha}=$ $\{3 / 4$.

$\left.\left[d^{\alpha(t o t)}\left(\sigma_{1}^{\alpha}\right)-d^{\alpha(t o t)}\left(\sigma_{2}^{\alpha}\right)\right]\right\}^{2 / 3}$. The coalescence of $\sigma_{1}^{\alpha}$ and $\sigma_{2}^{\alpha}$ corresponds to the observation point $\mathbf{x}$ crossing a surface, such that inside, each $\mathbf{x}$ is reached by four edge scattered rays, and outside by two. This type of surface is known as a smooth caustic. Note that $k_{\alpha} \mu_{\alpha}^{3 / 2}$ is proportional to the phase difference between two coalescing edge scattered rays. As usual, the Airy function $A i$ of $-k_{\alpha}^{2 / 3} \mu_{\alpha}$ describes the smooth caustic contribution. Inside a smooth $\alpha$-caustic boundary layer, the $\alpha$-component of the scattered field is $\mathbf{u}^{\alpha(\text { scat })}=\mathbf{u}^{\alpha(G E)}+\mathbf{u}_{1,2}^{\alpha(\text { caus })}+\sum_{m=3}^{4} \mathbf{u}_{m}^{\alpha(G T D)}$. The boundary layers surrounding non-smooth parts of the caustics where three $\sigma_{m}^{\alpha}$ coalesce may be described via the Pearcey integral or using numerical integration.

\section{Numerical results}

The code based on the time-harmonic asymptotic formulae has been fully tested against a numerical code based on the boundary integral equation method (Fig. 3). There is a good agreement for the range of parameters for which the applicability regions of both approaches overlap.

The time-harmonic asymptotics may be used to model the propagation of pulses by means of harmonic synthesis. Let us consider the following model parameters: the wave speeds $c_{P}=5840 \mathrm{~m} / \mathrm{s}, c_{S}=3170 \mathrm{~m} / \mathrm{s}$, the solid density $\varrho=7770 \mathrm{~kg} / \mathrm{m}^{3}$, and the pressure amplitude $P_{0}=1 \mathrm{MPa}$. For simplicity of presentation, let the pressure input be a narrow band pulse, 
(a)

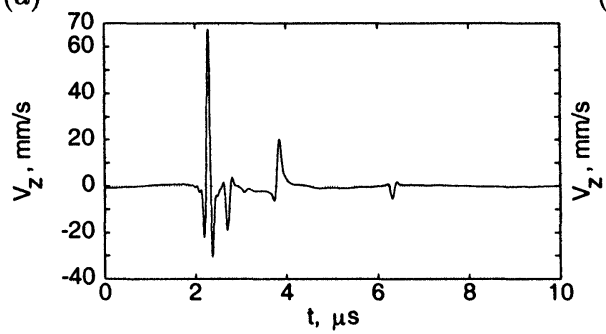

(b)

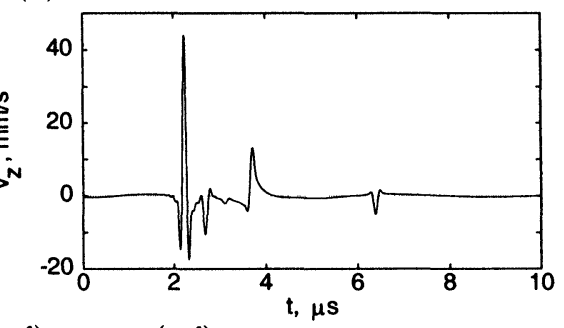

Figure 4. Representative velocity pulse trains, $\theta^{(\text {ref })}=30^{\circ}, \varphi^{(\text {ref })}=45^{\circ}, a=10 \mathrm{~mm}$, $b=5 \mathrm{~mm}$,

$r=15 \mathrm{~mm}, \varphi=45^{\circ}$, (a) $\theta=30^{\circ}$, (b) $\theta=75^{\circ}$, (c) $\theta=52^{\circ}$, (d)

$\theta=56^{\circ}$.

one cycle of $\sin (2 \pi f t), f=5 \mathrm{MHz}$. Typical pulse trains are presented in Fig. 4. In Fig. 4a the observation point with lies inside both $\mathrm{P}$ and $\mathrm{S}$ wave caustic surfaces. In Fig. $4 \mathrm{~b}$ the observation point lies outside both $\mathrm{P}$ and $\mathrm{S}$ caustics.

\section{Conclusions}

High-frequency asymptotic models for simulating ultrasonic pulse propagation and scattering in the transducer near have been developed. The models elucidate the physics of the problem and give explicit dependence on model parameters, thus allowing an easy prediction of pulse amplitudes and shapes. The corresponding asymptotic codes are at least hundred times faster than direct numerical codes, and practically just as accurate.

\section{Acknowledgements}

We thank Professor Borovikov for numerous discussions and Professor and Dr Glushkov for providing us with the boundary integral numerical code. The second author carried out this work at South Bank University as a Postdoctoral Fellow. He gratefully acknowledges the EPSRC Grant GR/L74750.

\section{References}

1. L Ju Fradkin, A P Kiselev, and E Krylova, 'Radiating Near Field Asymptotics of a Time-Harmonic Circular Normal Transducer in an Elastic Half-Space', J. Acoust. Soc. Am., Vol 104, No 3, pp 1178-1187, 1998. 
2. D Gridin, 'High-frequency asymptotic description of head waves and boundary layers surrounding the critical rays in an elastic half- space', J. Acoust. Soc. Am., Vol 104, No 3, pp 1188-1197, 1998.

3. D Gridin and L Ju Fradkin, 'High-Frequency Asymptotic Description of Pulses Radiated by a Circular Normal Transducer into an Elastic Half-Space', J. Acoust. Soc. Am., Vol 104, No 6, pp 3190-3198, 1998.

4. D Gridin, 'A fast method for simulating the propagation of pulses radiated by a rectangular normal transducer into an elastic half-space', J. Acoust. Soc. Am., Vol 104, No 6, pp 3199-3211, 1998.

5. D Gridin, 'On the radiation of ultrasound into an isotropic elastic half-space via wave-front expansions of the impulse response', J. Acoust. Soc. Am., Vol 105, No 5, pp 2565-2573, 1999.

6. D Gridin, 'The radiating near field of a circular transducer of arbitrary apodization on an elastic half-space', J. Acoust. Soc. Am., Vol 106, No 3, 1999.

7. J D Achenbach, A K Gautesen and H McMaken, Ray Methods for Waves in Elastic Solids; With Applications to Scattering by Cracks (Pitman, N. Y., 1982).

8. H McMaken, 'A Uniform Theory of Diffraction for Elastic Solids', J. Acoust. Soc. Am., Vol 75, No 5, pp 1352-1359, 1984.

9. V A Borovikov, Uniform Stationary Phase Method (IEE, London, 1994).

10. R Wong, Asymptotic approximations of integrals (Academic Press, N. Y., 1989).

11. V A Borovikov and B Ye Kinber, Geometrical Theory of Diffraction (IEE, London, 1994).

\section{Appendix}

\section{The Fourier Amplitudes in the Canonical Problem}

The Fourier transforms in (5) involve analytical functions in the upper $\xi$ half-plane which are given by the following formulas from Achenbach ${ }^{7}$ (Sec. 5.3) where somewhat different notations are used:

$$
\begin{aligned}
A_{0}^{+}(\xi) & =\frac{\left(k_{S}\right)^{2}-2\left(\xi^{2}+\eta^{2}\right)}{i\left(k_{S}\right)^{2}} U_{1}\left(\xi, \xi_{0}, \eta\right) \\
A_{0}^{-}(\xi) & =-\frac{2}{i k_{S}}\left[\xi U_{3}\left(\xi, \xi_{0}, \eta\right)-\eta U_{2}\left(\xi, \xi_{0}, \eta\right)\right], \\
A_{1}^{+}(\xi) & =\frac{\xi \eta U_{2}\left(\xi, \xi_{0}, \eta\right)+\left[\gamma_{S}^{2}(\xi)-\xi^{2}\right] U_{3}\left(\xi, \xi_{0}, \eta\right)}{i\left(k_{S}\right)^{2}} \\
A_{1}^{-}(\xi) & =\frac{2}{i k_{S}} \xi U_{1}\left(\xi, \xi_{0}, \eta\right)
\end{aligned}
$$




$$
\begin{aligned}
& A_{2}^{+}(\xi)=\frac{\left[\eta^{2}-\gamma_{S}^{2}(\xi)\right] U_{2}\left(\xi, \xi_{0}, \eta\right)-\xi \eta U_{3}\left(\xi, \xi_{0}, \eta\right)}{i\left(k_{S}\right)^{2}} \\
& A_{2}^{-}(\xi)=\frac{2}{i k_{S}} \eta U_{1}\left(\xi, \xi_{0}, \eta\right) \\
& A_{3}^{+}(\xi)=0, \\
& A_{3}^{-}(\xi)=-\frac{1}{i k_{S}}\left[\xi U_{2}\left(\xi, \xi_{0}, \eta\right)+\eta U_{3}\left(\xi, \xi_{0}, \eta\right)\right] .
\end{aligned}
$$

The functions $\left.U_{1}(\xi), U_{2}(\xi)\right)$ and $U_{3}(\xi)$ are defined by

$$
\begin{gathered}
{\left[\begin{array}{l}
U_{1}(\xi) \\
U_{2}(\xi)
\end{array}\right]=i\left[\begin{array}{cc}
-\eta d(\xi) & -\xi c(\xi) \\
\xi d(\xi) & -\eta c(\xi)
\end{array}\right]\left\{C \frac{\xi+\xi^{i n c}}{\xi^{2}+\eta^{2}}\left[\begin{array}{l}
1 \\
g
\end{array}\right]\right.} \\
\left.+\frac{1}{(\xi-i|\eta|)\left(\xi^{i n c}-i|\eta|\right)}\left[\begin{array}{c}
-d\left(\xi^{i n c}\right)\left(\eta t_{1}+\xi^{i n c} t_{2}\right) \\
c\left(\xi^{i n c}\right)\left(\eta t_{2}-\xi^{i n c} t_{2}\right)
\end{array}\right]\right\} \\
U_{3}(\xi)=\frac{i t_{3} \sqrt{q^{P}+\xi} \sqrt{q^{P}-\xi^{i n c}}}{\kappa^{2}\left(q^{R}+\xi\right)\left(q^{R}-\xi^{i n c}\right) K(\xi) K\left(\xi^{i n c}\right)}
\end{gathered}
$$

where $q^{\alpha}=\sqrt{\left(k_{\alpha}\right)^{2}-\eta^{2}} ; k_{R}$ is the Rayleigh wave number; $\kappa=\sqrt{2\left(\left(k_{P}\right)^{2} /\left(k_{S}\right)^{2}\right)-1}$ $C=-2 i|\eta|\left[g c\left(\xi^{i n c}\right)\left(\eta t_{2}-\xi^{i n c} t_{1}\right)-d\left(\xi^{i n c}\right)\left(\eta t_{1}+\xi^{i n c} t_{2}\right)\right] /\left(1-g^{2}\right)\left[\left(\xi^{i n c}\right)^{2}+\eta^{2}\right]$; with

$$
\begin{array}{ll}
c(\xi)=\frac{1}{\sqrt{q^{S}+\xi}}, & d(\xi)=\frac{\sqrt{q^{S}+\xi}}{i \kappa\left(q^{R}+\xi\right) K(\xi)} \\
g=\operatorname{sgn}(\eta) \frac{\kappa\left(q^{R}+i|\eta|\right) K(i|\eta|)}{q^{S}+i|\eta|}, & K(\xi)=\exp \left(-\frac{1}{\pi} \int_{q^{P}}^{q^{s}} \frac{\tan ^{-1}\left[a_{1}(t) / a_{2}(t)\right]}{t+\xi} d t\right)
\end{array}
$$

where correcting an obvious misprint in [7], Eq. 5.B.5, the $a$-functions are $a_{1}(t)=4\left(t^{2}+\eta^{2}\right) \sqrt{\left(q^{S}\right)^{2}-t^{2}} \sqrt{t^{2}-\left(q^{P}\right)^{2}}$ and $a_{2}(t)=\left[2\left(\eta^{2}+t^{2}\right)-\left(k_{S}\right)^{2}\right]^{2}$. Finally, we have

$$
\begin{aligned}
t_{1} & =-2 \cos \theta^{i n c} \mathbf{k}^{P(\text { inc })} \cdot \mathbf{e}_{x}, \\
t_{2} & =-2 \cos \theta^{i n c} \mathbf{k}^{P(\text { inc })} \cdot \mathbf{e}_{y}, \\
t_{3} & =\left[\left(k_{S}\right)^{2}-2\left(k_{P}\right)^{2} \sin ^{2} \theta^{i n c}\right] k_{P} .
\end{aligned}
$$

Note that using [7], Eqns. (5.39), (5.40) and (5.41) the following formula holds

$$
\begin{aligned}
& K(\xi) K(-\xi)= \\
& \left.2\left(\left(k_{S}\right)^{2}-\left(k_{P}\right)^{2}\right)\left[\gamma_{S}(\xi)^{2}-\left(\eta^{2}+\xi^{2}\right)\right]^{2}+4\left(\xi^{2}+\eta^{2}\right)^{2} \gamma_{P}(\xi) \gamma_{S}(\xi)\right] / \gamma^{R}(\xi) .
\end{aligned}
$$

Also, when the crack is elliptic the underlying coordinate system is local rather than Cartesian. 


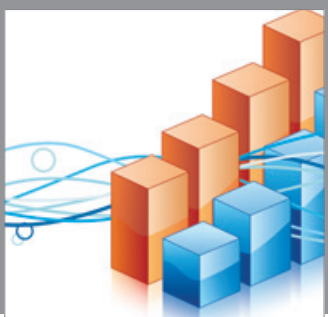

Advances in

Operations Research

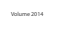

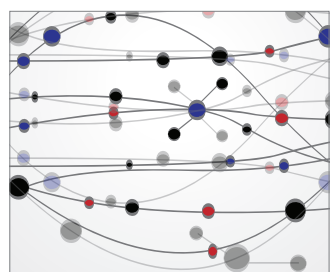

\section{The Scientific} World Journal
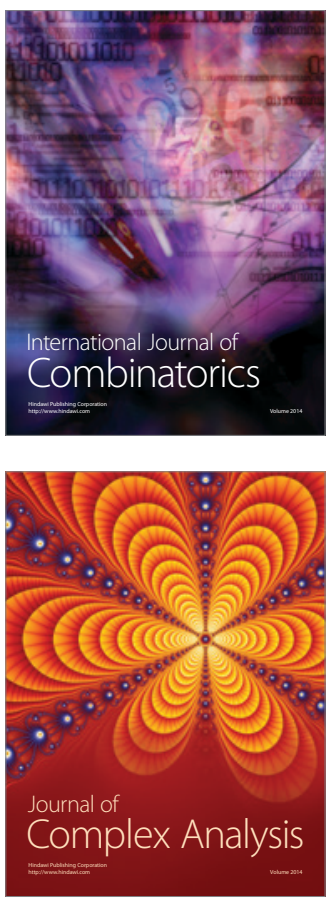

International Journal of

Mathematics and

Mathematical

Sciences
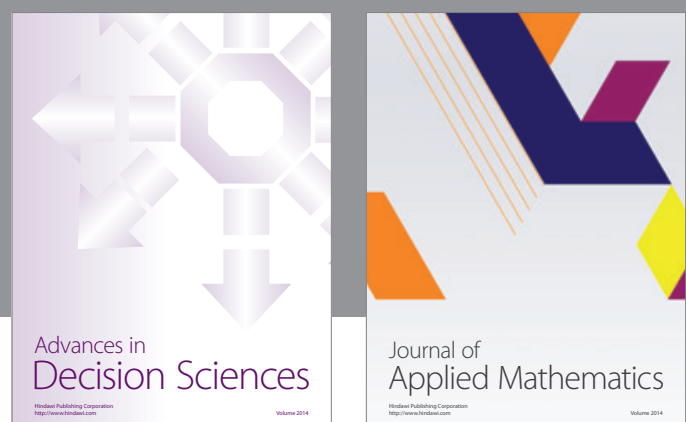

Journal of

Applied Mathematics
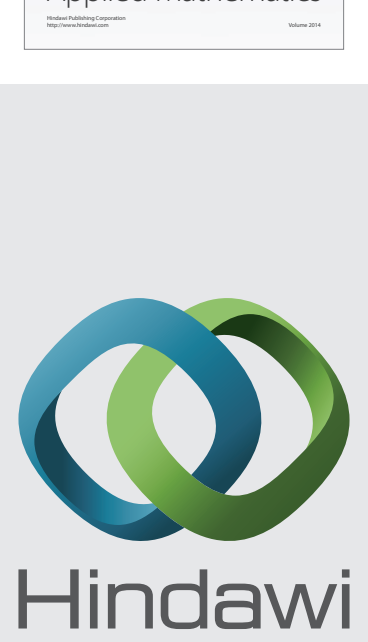

Submit your manuscripts at http://www.hindawi.com
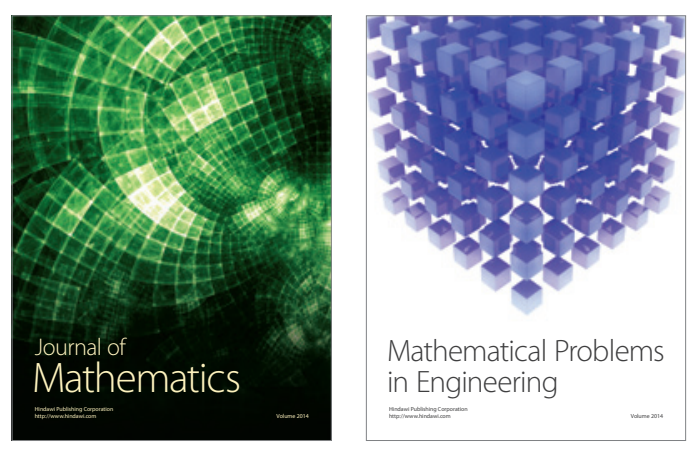

Mathematical Problems in Engineering
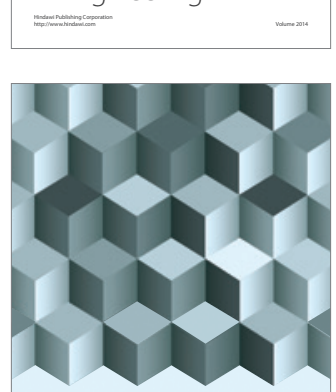

Journal of

Function Spaces
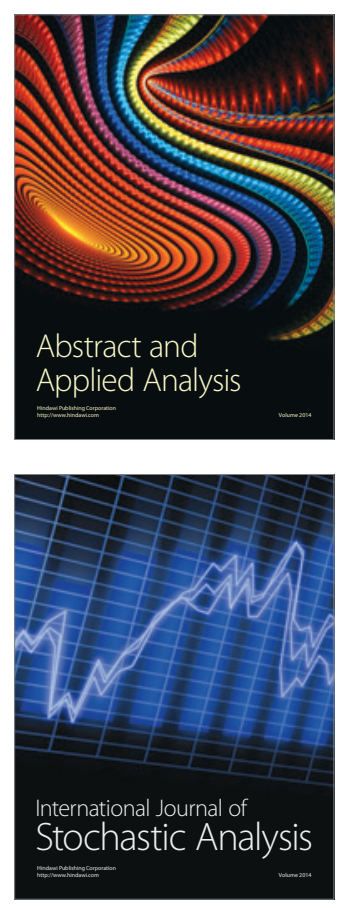

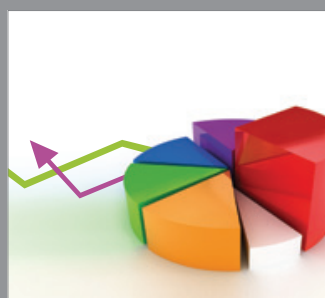

ournal of

Probability and Statistics

Promensencen
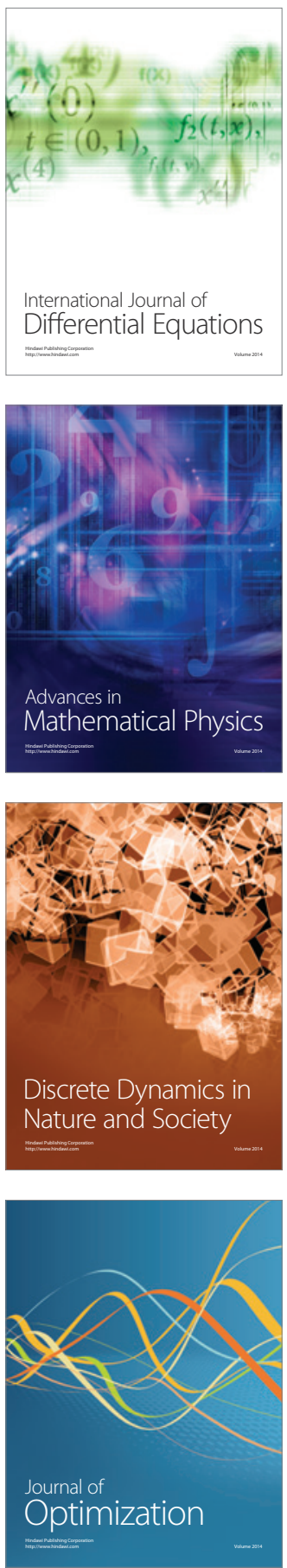